\section{LUPUS SCIENCE\& MEDICINE}

To cite: Concha JSS, Werth VP. Alopecias in lupus erythematosus. Lupus Science \& Medicine 2018;5:e000291. doi:10.1136/ lupus-2018-000291

Received 4 July 2018 Revised 15 August 2018 Accepted 2 September 2018
Check for updates

(C) Author(s) (or their employer(s)) 2018. Re-use permitted under CC BY-NC. No commercial re-use. See rights and permissions. Published by BMJ.

${ }^{1}$ Corporal Michael J Crescenz VAMC, Philadelphia, Pennsylvania, USA

2Department of Dermatology, Perelman School of Medicine at the University of Pennsylvania, Philadelphia, Pennsylvania, USA

Correspondence to Dr Josef Symon Salgado Concha; Josef.Concha@uphs. upenn.edu

\title{
Alopecias in lupus erythematosus
}

\author{
Josef Symon Salgado Concha, ${ }^{1,2}$ Victoria P Werth ${ }^{1,2}$
}

\begin{abstract}
Several patterns of hair loss can occur in lupus erythematosus (LE). Alopecias which show histological characteristics of LE are LE-specific, and include discoid LE (DLE), diffuse or patchy hair loss in acute LE, subacute cutaneous LE, and rarely tumid LE. Lupus hair in SLE is a poorly characterised entity and may be a form of telogen effluvium. Alopecia areata can coexist with LE and may mimic DLE. Non-lupus alopecias such as telogen effluvium and anagen effluvium have a myriad of causes which include disease flares, drugs and stress in the setting of LE. The latest validated Systemic Lupus International Collaborating Clinics classification criteria for SLE includes non-scarring alopecia as a criterion; therefore, recognising the aetiology of hair loss in the setting of LE is crucial in classifying a patient to have systemic disease.
\end{abstract}

\section{INTRODUCTION}

Lupus erythematosus (LE) is a chronic multiorgan autoimmune disease with a spectrum of clinical and serological presentations. ${ }^{1-3}$ The major target organs are the joints, skin, kidneys, lungs, and the nervous and serous systems, with ANA as the frequent hallmark antibody. ${ }^{2}{ }^{4}$ At any point during the disease course of SLE, dermatological findings may be found in over $80 \%$ of patients. ${ }^{4-7}$ Certain presentations of LE on the skin and hair can aid in assessing, classifying and predicting systemic involvement. $^{4-10}$

Hair loss is a frequent occurrence in SLE and is present in more than half of the patients at some point during the course of the disease. ${ }^{811-14}$ Although several patterns of hair loss can exist in the setting of SLE, the aetiology is not always specific to LE (box 1). Determining whether alopecia is inherent to $\mathrm{LE}$ or just coincidental to $\mathrm{LE}$ is crucial because it has been included in several classification systems for SLE (table 1), including the latest Systemic Lupus International Collaborating Clinics (SLICC) classification criteria. ${ }^{1}$ Non-scarring alopecia, in particular, has been incorporated in the SLICG criteria because its specificity to SLE is high (95.7) in the derivation sample, and it met the standards of clinical consensus among experts. ${ }^{12}$ Non-scarring alopecia is clinically defined by the SLICC as diffuse thinning and fragility of the hair in the absence of other causes. ${ }^{1}$ Several processes that result in non-scarring alopecia must therefore be ruled out before attributing hair loss to LE (boxes 1 and 2).

In this paper, we discuss an approach to recognising the different causes of hair loss that occur in LE and their differential diagnoses. The categorisation we employ is largely based on how scalp biopsy features are consistent with the diagnosis of LE. We also expand on the alternate diagnoses of non-scarring alopecia in LE. Certain points in the history and physical exam (which may necessitate the use of dermoscopy) can, in the majority of cases, lead the physician to make a confident diagnosis. However, non-scarring alopecia in SLE has a wide range of differential diagnoses (boxes 1 and 2) which can challenge a physician's clinical acumen. In a patient suspected to have SLE but with an unclear aetiology of hair loss, working closely with dermatologists and performance of ancillary tests such as a scalp biopsy, direct immunofluorescence (DIF) and/or serological markers may be warranted.

\section{OVERVIEW OF ALOPECIAS IN LE}

LE-specific alopecia is classically exemplified by scalp discoid lupus erythematosus (DLE), which is typically scarring. ${ }^{13-16}$ Several non-scarring forms of hair loss such as lupus hair, alopecia areata and telogen/anagen effluvium do not display biopsy features of LE and are considered either LE non-specific or just coincidental with LE. ${ }^{14-16}$ Subacute cutaneous lupus erythematosus (SCLE), tumid LE and acute LE display histopathological features consistent with LE and are lupus-specific alopecias. ${ }^{17-24}$ However, attribution is not always straightforward because LE-specific alopecias can coexist with the more common causes of hair loss and can possess similar clinical features. ${ }^{14} 19$ 25-27 Moreover, it should be kept in mind that certain cutaneous findings on the scalp (DLE, SCLE, diffuse non-scarring hair loss due to acute LE) are distinct items in the classification criteria for SLE and may count as separate items in fulfilling the criteria (table 1$)$. 


\section{Box 1 Alopecias in lupus erythematosus}

\section{Lupus-specific alopecia.}

- Discoid lupus erythematosus. *

- Acute lupus erythematosus. $†$

- Subacute cutaneous lupus erythematosus. $\dagger$

- Tumid lupus erythematosus. $\dagger$

Lupus non-specific alopecia.

- Lupus hair. $\dagger$

Alopecia areata/ophiasis. $\dagger$

Non-lupus alopecia.

- Telogen effluvium. $\dagger$

- Anagen effluvium. $\dagger$

*Non-scarring in its early stage.

†Typically non-scarring.

\section{LUPUS-SPECIFIC ALOPECIA \\ DLE of the scalp}

The scarring alopecia of DLE is an LE-specific cutaneous lesion according to Gilliam's classification of cutaneous LE. ${ }^{28}$ DLE is a cutaneous item in the SLICC criteria and is counted separately from non-scarring alopecia. ${ }^{1}$

\begin{tabular}{lll}
\hline $\begin{array}{l}\text { Table 1 } \\
\text { features }^{12}\end{array}$ & SLE criteria throughout the years with cutaneous \\
\hline Criteria & Criteria items & $\begin{array}{l}\text { Alopecia as a } \\
\text { criterion }\end{array}$ \\
\hline 1971 ACR & $\begin{array}{l}6 \text { cutaneous items } \\
\text { (malar rash, discoid } \\
\text { rash*, Raynaud's } \\
\text { phenomenon, alopecia, } \\
\text { photosensitivity, oral/ } \\
\text { nasopharyngeal ulcers). }\end{array}$ & $\begin{array}{l}\text { Rapid loss of a large } \\
\text { amount of scalp } \\
\text { hair, by patient's }\end{array}$ \\
observation.†
\end{tabular}

${ }^{*}$ Can also present clinically as alopecia.

†Definition does not require histopathological/immunopathological confirmation.

ACR, American College of Rheumatology; SLICC, Systemic Lupus International Collaborating Clinics.

\section{Box 2 Differential diagnoses of alopecias}

\section{Scarring alopecias.}

- Lichen planopilaris.

- Frontal fibrosing alopecia.

- Central centrifugal cicatricial alopecia.

> Pseudopelade of Brocq.

- Tinea capitis (late stage).

Non-scarring alopecias.

- Patterned hair loss.

- Acute diffuse and total alopecia areata.

- Trichotillomania.

- Syphilitic alopecia.

Tinea capitis (early stage).

\section{Epidemiology}

Scalp is the most common site affected by DLE, occurring in $30 \%-50 \%$ of reported cases ${ }^{29-31}$ In its early stages, DLE lesions are only slightly inflammatory and non-scarring. ${ }^{25}$ However, as much as $60 \%$ of patients with scalp DLE continue to progress towards permanent and irreversible hair loss. ${ }^{32}$ It accounts for $30 \%-40 \%$ of all scarring alopecias combined ${ }^{33-35}$ and about $60 \%$ of all primary lymphocytic alopecias, making it the most common scarring alopecia in this subgroup. ${ }^{14}{ }^{33}$ In a prospective cross-sectional study, the proportion of patients with scarring alopecia did not differ across LE subgroups (generalised DLE, localised DLE, systemic LE) ${ }^{31}$ Although scalp DLE is more frequent in women, ${ }^{31} 3235$ there is an equal proportion of both sexes in terms of the tendency to develop scarring alopecia from DLE. ${ }^{31}$ The age of onset is usually between 20 and 40 years of age, ${ }^{14} 303335$ and it is reported to be most prevalent among African-Americans. ${ }^{30} 32$ The presence of scarring alopecia in a patient with DLE portends a prolonged disease course. ${ }^{141}$

\section{Pathology}

The hallmark finding in cicatricial alopecias is fibrosis of the follicular structure, which is appreciated clinically as obscured follicular openings on the scalp. ${ }^{36}$ The scarring process usually begins with the inflammatory cells infiltrating the follicular bulge, a contiguous part of the outer root sheath located at the insertion point for the arrector pili muscle. This marks the bottom of the permanent portion of hair follicles and is also where the epidermal hair stem cells are located. ${ }^{14}$ Once replacement by fibrous tissue is established, hair loss is permanent and irreversible. ${ }^{3637}$ Scarring alopecias are usually either (1) primary, in which the hair follicle is the main target of inflammation; or (2) secondary, if a separate disease process causes destruction of the nearby follicular structure ${ }^{34}$ In 2001, the North American Hair Research Society proposed a classification scheme for the primary scarring alopecias based on the predominant inflammatory cell type on histology (lymphocytic, neutrophilic, mixed, non-specific).$^{36}$ Scalp DLE serves as a model for 
studying the processes leading to primary lymphocytic cicatricial alopecias. ${ }^{37}$

Although the pathogenesis of scarring in DLE is not fully elucidated, several hypotheses can explain the processes leading to the collagenous transformation of the follicular structure in primary cicatricial alopecias. ${ }^{3436} 37$ In scalp DLE, the most likely determinant of fibrosis is the aggregation of inflammatory infiltrates in the bulge area of the follicle where there is a high concentration of antigen-presenting Langerhans cells in close association with epithelial hair follicle stem cells. ${ }^{14} 37$ The aggregation of inflammatory infiltrates around the pilosebaceous junction also leads to the destruction of the sebaceous gland in DLE, ${ }^{14}$ a feature that is not usually present in acute or subacute cutaneous LE. ${ }^{31}$ When there is no sebum to regulate the outward movement of the hair, the shaft moves proximally perforating the bulbar region and inciting a foreign body granulomatous reaction that precipitates the scarring process. ${ }^{34}$ DLE lesions of the scalp are susceptible to Koebnerisation, increasing in number on sites frequently rubbed and scratched such as the scalp. ${ }^{14}$ Although ultraviolet light is a known trigger of cutaneous lupus erythematosus (CLE), interestingly, in one prospective cross-sectional study, sun-exposed alopecic patches of patterned hair loss and sites with prominent solar elastosis did not show an increased tendency to develop discoid lesions. ${ }^{31}$ Smoking has been found to worsen DLE on the scalp and to make the lesions more resistant to therapy. ${ }^{38}$

\section{Clinical features}

A panel of international experts recently proposed the following morphological features to distinguish DLE from other mimickers: (1) an erythematous to violaceous hue, (2) features of damage such as atrophic scarring and dyspigmentation, (3) follicular hyperkeratosis or plugging, and (4) the presence of scarring alopecia. ${ }^{39}$ In addition, telangiectasia and scale are also frequently present. ${ }^{1433}$ These changes are more prominent on the centre of the alopecic patch in contrast to other cicatricial alopecias in which clinical activity is more prominent on the peripheral rim (figure 1A) ${ }^{30}$ Hair on these sites are usually brittle and easily detachable after a hair pull test. ${ }^{2735}$ Lesions are more likely to be multiple than solitary, and are confined most commonly on the vertex..$^{33}$ DLE on the scalp can be itchy, tender or burning, but sometimes they produce no symptoms. ${ }^{14}$

\section{Diagnosis}

Dermoscopy has recently been found to approach a specificity of $100 \%$ in the diagnosis of DLE when features such as tortuous blood vessels and follicular keratotic plugs are seen (figure 1B).$^{40}$ Follicular plugging can be a feature of both acute and chronic alopecic lesions. ${ }^{29}$ Another very specific dermoscopic finding is the presence of follicular red dots, which correspond to multiple widened infundibula bordered by ectatic vessels and extravasated red blood cells. ${ }^{41}$

The North American Hair Research Society recommends performing at least one $4 \mathrm{~mm}$ punch biopsy down to the subcutaneous tissue in the area of active hair loss. ${ }^{36}$ The HoVert technique obtains both horizontal and vertical sections in a single punch biopsy specimen. ${ }^{42}$ A horizontal section allows for visualisation of follicular units, whereas a vertical section exhibits the dermoepidermal junction and may be used for immunofluorescence studies. ${ }^{36}$ Other optional but recommended stains include elastin, mucin or colloidal iron, Gram, and periodic acid-Schiff. ${ }^{34}{ }^{36}$ If infection needs to be ruled out, histopathology samples may be sent for cultures. ${ }^{36}$ The most characteristic histopathological features of DLE are interface dermatitis (basal cell vacuolisation, apoptotic keratinocytes and obscuring of the dermoepidermal junction with inflammatory cells), lymphohistiocytic infiltration around the vessels and appendages, follicular keratotic plugs, mucin deposition, and basement membrane thickening. ${ }^{33} 39$ Later

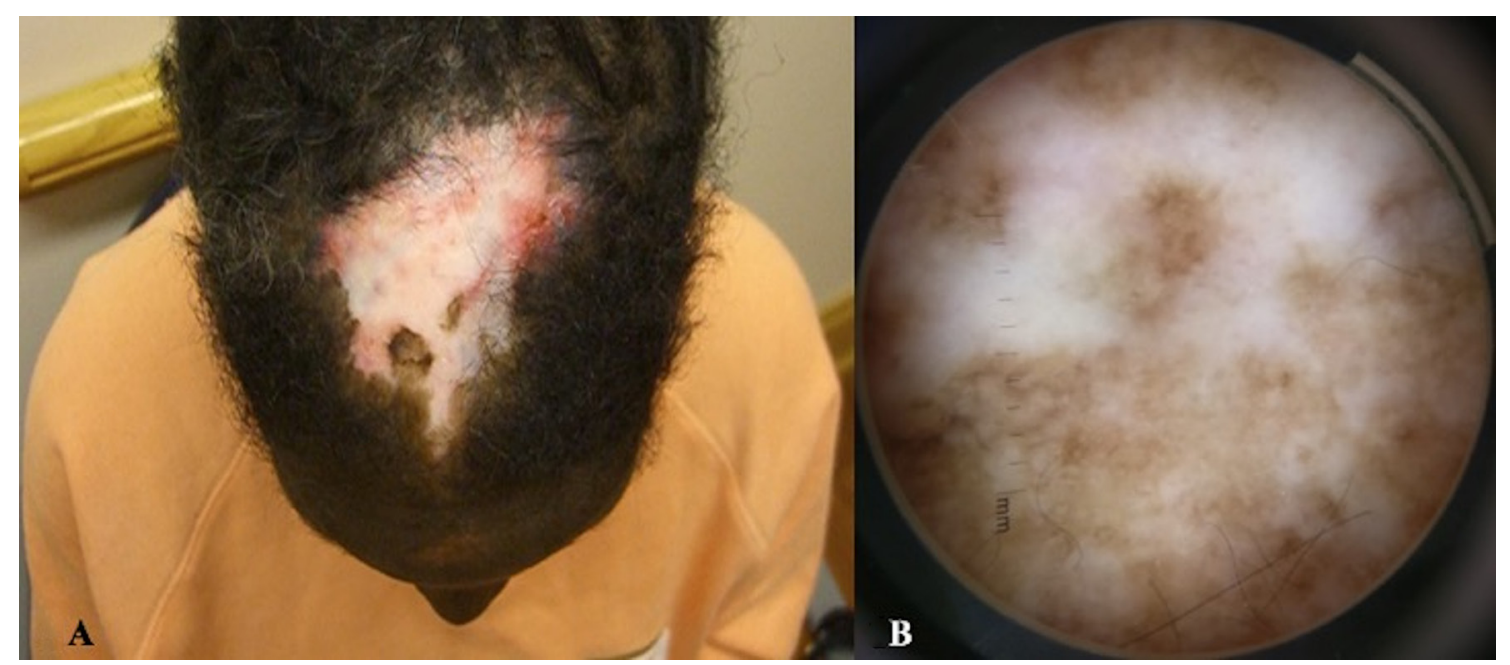

Figure 1 (A) Discoid lupus erythematosus. An atrophic porcelain-white plaque on the vertex with areas of erythema, scale, dyspigmentation on the periphery. (B) Dermoscopy of discoid lupus erythematosus. Loss of follicular ostia, areas of follicular plugging, dyspigmentation and pinpoint follicular red dots. 
stages of the disease show reduced follicular units and interstitial fibrosis. ${ }^{30} 34$

Immunopathology can aid in the correct diagnosis when histopathology does not show the usual distinguishing features of DLE. ${ }^{33}$ DIF of lesional skin can differentiate DLE from mimickers such as lichen planus or lichen planopilaris, approaching a specificity of 0.97 , which is higher than histopathology alone $(0.84){ }^{43}$ The lupus band test, described as granular deposition of immunoglobulin and complement deposition at the dermoepidermal junction (), can be positive in as high as $81.7 \%$ of patients with scalp DLE. ${ }^{33}$

ANA positivity among patients with scalp DLE ranges from $15 \%$ to $42 \%$ of cases. ${ }^{33-35}$ Hence, it is reasonable to order a complete blood count, urinalysis with urine protein, ANAs and extractable nuclear antigens in a patient with scalp DLE. ${ }^{34} 44$

\section{Differential diagnoses of DLE (other scarring alopecias) Lichen planopilaris}

Lichen planopilaris (LPP) is the most common differential diagnosis made by dermatologists in patients suspected with scalp DLE. ${ }^{32}$ LPP classically presents as violaceous glistening, flat-topped polygonal papules with adherent fine white scales (Wickham's striae) on the scalp similar to lichen planus (LP) ${ }^{45}$ However, most of the time, only erythematous plaques with variable degrees of scarring, ulceration and/or atrophy are seen on clinical inspection. ${ }^{35}$ In contrast to DLE, LP of the scalp presents with perifollicular erythema, less dyspigmentation and a more intense pruritus. ${ }^{145}$ Another helpful clinical clue is the presence of acuminate perifollicular keratotic plugs on the periphery of the alopecic patches which is unlike the follicular plugging seen on the centre of the alopecic patches in scalp DLE. ${ }^{27}$ Remaining hairs on these patches are of the anagen type and can be easily pulled out. ${ }^{46}$ In $50 \%$ of cases, there are lichenoid lesions on sites other than the scalp. ${ }^{47}$ It is recommended to perform a biopsy on the margin of the alopecic patch where the disease process is most active. ${ }^{14}$ Histopathological features during the early stage of the disease resemble those seen in classic LP. ${ }^{14} 343546$ Hyperkeratosis, acanthosis, hypergranulosis, an interface lichenoid pattern confined around the infundibulum and isthmus, and perifollicular lamellar fibrosis are the usual features. ${ }^{14} 3435$ Mucin stain can highlight the deposition of glycosaminoglycans in the perifollicular areas, in contrast to the deep and interstitial involvement in DLE. ${ }^{32}{ }^{47}$ However, histopathology is not definitive in differentiating LP from DLE especially in the late stage of the disease when only fibrotic tracts remain. ${ }^{40}{ }^{46}$ Dermoscopy can further aid in identifying unique LP features such as tubular desquamation, which is known to be most characteristic. ${ }^{464}{ }^{49}$ DIF is non-specific and may show immunoglobulin staining (especially IgM) of colloid bodies along the upper portion of the hair follicle. ${ }^{14}{ }^{34}$ There are a number of case reports of scalp LE/LP overlap in which the histopathological findings show LE, but the lupus band test is negative and DIF

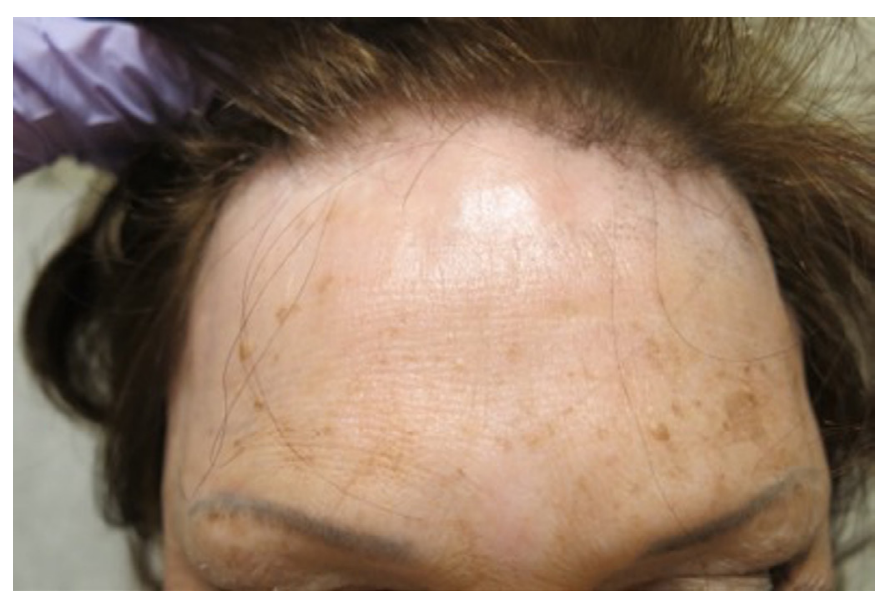

Figure 2 Frontal fibrosing alopecia. Receding hairline on the frontal scalp, with bilateral loss of eyebrows.

shows features of LP. ${ }^{45}{ }^{50}$ LPP is infrequently associated with hepatitis B or C infection; therefore, viral serologies and liver function tests may be obtained (box 2). ${ }^{34}$

\section{Frontal fibrosing alopecia}

Frontal fibrosing alopecia (FFA) is a special variant of LP presenting as progressive scarring hair loss in a bandlike distribution along the frontotemporal scalp mostly affecting postmenopausal women (figure 2). ${ }^{1451-53}$ Aside from the recession of the frontal hairline, half of these patients suffer from eyebrow alopecia. ${ }^{34}$ Other associated clinical features are facial papules, erythematous macules on the glabella and loss of body hair. ${ }^{51}$ FFA shares the same histological and immunopathological features with LPP, but it additionally involves the intermediate and miniaturised (vellus hair) follicles. ${ }^{34} 47$ FFA has been reported to coexist with DLE; therefore, the presence of cutaneous lesions of LP or LE elsewhere does not entirely rule out DLE. ${ }^{5152}$ Conversely, prior diagnosis of CLE does not preclude the diagnosis of LP scarring alopecia. ${ }^{52}$

\section{Central centrifugal cicatricial alopecia}

The classic presentation of central centrifugal cicatricial alopecia (CCCA) is a centrifugally expanding area of scleroatrophic alopecia on the crown or vertex most commonly seen among middle-aged African-American women. ${ }^{1427} 3447$ The non-inflammatory centre of the plaque has increased interfollicular spacing due to a mixture of tufted and normal-appearing hairs. ${ }^{14} 47 \mathrm{~A}$ burning, tender or itching sensation is commonly felt on the active sites. ${ }^{14} 27$ The pathogenesis is poorly understood, but is usually a combination of genetics and certain traumatic hair care practices. ${ }^{27} 34 \mathrm{~A}$ frequently cited distinguishing histopathological feature of CCCA is the premature desquamation of the inner root sheath. ${ }^{14}{ }^{47} \mathrm{~A}$ retrospective review has recently shown that the presence of a peripilar white grey halo surrounding the emerging hairs on dermoscopy is highly specific and sensitive for CCCA. ${ }^{54}$ In contrast to DLE, there is no interface dermatitis and follicular plugging, and DIF is negative. ${ }^{47}$ 


\section{Pseudopelade of Brocq}

Pseudopelade of Brocq (PB) is characterised by non-inflammatory, ivory-coloured, scleroatrophic patches on the scalp resembling 'footprints in the snow'. 33354755 This controversial clinical phenotype of scarring alopecia is considered by some to be a form of dermal atrophy rather than a true cicatricial process. ${ }^{334}$ By definition, the onset is non-inflammatory and insidious, beginning with few flesh-toned macules coalescing into larger smooth and shiny depressed patches. ${ }^{26}$ This entity is considered by some to be a diagnosis of exclusion. ${ }^{33} 35$ Immunopathology should be negative. ${ }^{35} 47$ Interestingly, in a cohort of patients with scalp DLE, 30\% showed clinical features consistent with $\mathrm{PB}$, but histopathology and DIF showed features consistent with DLE. ${ }^{56}$

\section{Miscellaneous}

DLE can initially present as round, non-inflammatory patches of hair loss resembling alopecia areata. ${ }^{16} 2557$ Head and neck cancers that are treated with chronic high dose of radiation can later on develop within the irradiated skin well-demarcated, poikilodermatous, scarring plaques resembling chronic CLE. ${ }^{14}$ Folliculitis decalvans classically presents with an advancing alopecic patch on the vertex of the scalp surrounded by pustules and perifollicular papules, but its chronic stage can resemble burned-out lesions of DLE. ${ }^{16}$ Long-standing DLE lesions that ulcerate and bleed should be evaluated for squamous cell carcinoma (SCC) because this malignancy has a high metastatic rate in this setting. ${ }^{14}$ Clinical characteristics that necessitate close follow-up for the development of SCC are male sex, tobacco use, lip involvement, early onset and treatment resistance.$^{58}$ A patient with SLE presenting with non-pruritic, scaly erythematous plaques on the scalp was diagnosed with psoriatic alopecia after correlating the clinical, dermoscopic and histopathological findings. ${ }^{59}$ Erythematous scarring plaques with dyspigmentation and atrophy on the scalp of an AfricanAmerican patient were initially treated as DLE, but were eventually diagnosed as pemphigoid after histopathology and DIF were performed ${ }^{60}$ Sarcoidal alopecia and scalp DLE have similar clinical and dermoscopic features, and can only be differentiated by histopathological examination. ${ }^{61}$

\section{LE-specific alopecia in the setting of acute LE}

This type of hair loss in LE may be confused with telogen effluvium, anagen effluvium or alopecia universalis (figure 3).${ }^{152}$ However, this is considered a distinct entity because it shows interface dermatitis, and obscuring of the dermoepidermal junction by inflammatory cells on histology and a positive lupus band test. ${ }^{18}$ Some suggest that its clinical presentation more likely fits the non-scarring early stage of DLE, rather than a separate form of LE-specific alopecia. ${ }^{25}$ In addition, histological findings of early DLE may be similar to those seen in acute and chronic CLE. ${ }^{30}{ }^{63}$ However, points which do not support the diagnosis of DLE include (1) a diffuse pattern,

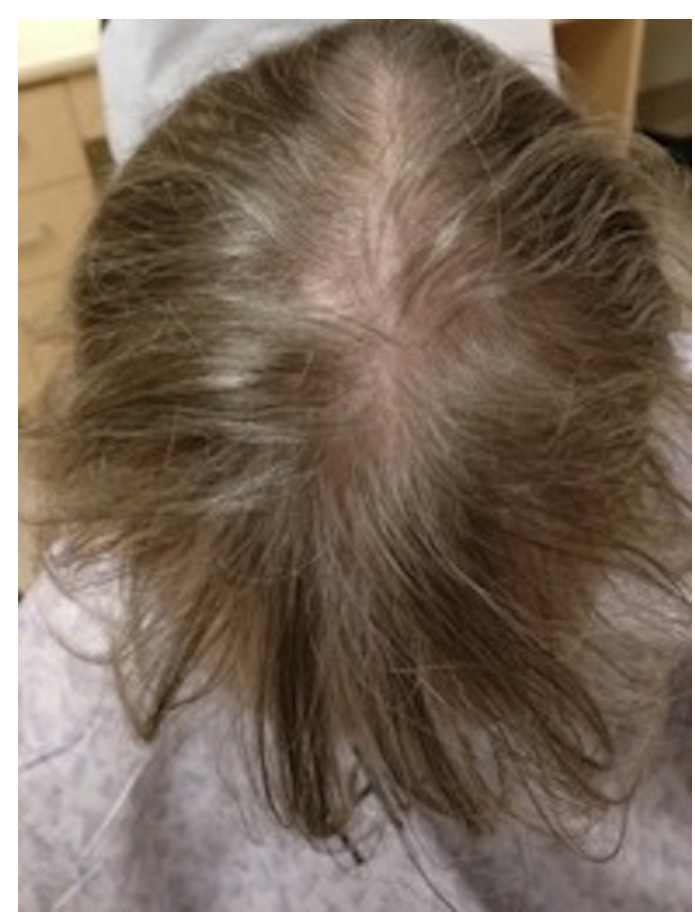

Figure 3 Diffuse hair loss in a patient with acute lupus erythematosus. Alopecia resembles patterned hair loss, but occurs in the setting of a lupus erythematosus flare.

(2) absence of follicular plugging and dyschromia on trichoscopy, (3) serology and immunofluorescence tests favouring SLE, and (4) full regrowth of hair after immunosuppressive therapy. ${ }^{19}$ Establishing the correct diagnosis in this scenario is important in terms of disease classification because the SLICC criteria include both DLE and non-scarring alopecia as clinical items to diagnose SLE, whereas the 1997 American College of Rheumatology criteria only include discoid rash. ${ }^{2}$ Non-scarring diffuse alopecia in SLE usually reflects the underlying disease activity or severity, with improvement in hair growth once an acute LE flare abates. ${ }^{814} 161864$

A patchy type of alopecia is also a relatively frequent manifestation in SLE occurring in about $14 \%-50 \%$ of patients. ${ }^{1724}$ These multiple partially alopecic patches can occur before, during or after the diagnosis of SLE, ${ }^{24}$ although it is commonly associated with severe established SLE. ${ }^{15} 1624$ Remaining within these patches are fractions of telogen or dystrophic anagen hairs. ${ }^{16}$ It is believed by some authors that cases previously clinically diagnosed as alopecia areata in the setting of lupus are actually patchy LE hair loss. ${ }^{17}$ In LE, areas of incomplete hair loss have some degree of erythema within the patch, and dermoscopy may reveal hair shaft hypopigmentation and vessel changes. ${ }^{15} 17$ Histopathological features that support the diagnosis of LE-specific patchy alopecia are atrophy of the epidermis, hyperkeratosis, interface dermatitis of the epidermal basal cells and pigment incontinence. ${ }^{17}$ However, in some cases, histopathology alone cannot distinguish LE from AA or even syphilitic alopecia; therefore, further testing such as DIF and serologies should be performed. ${ }^{15172465}$ One recommendation is to order an 


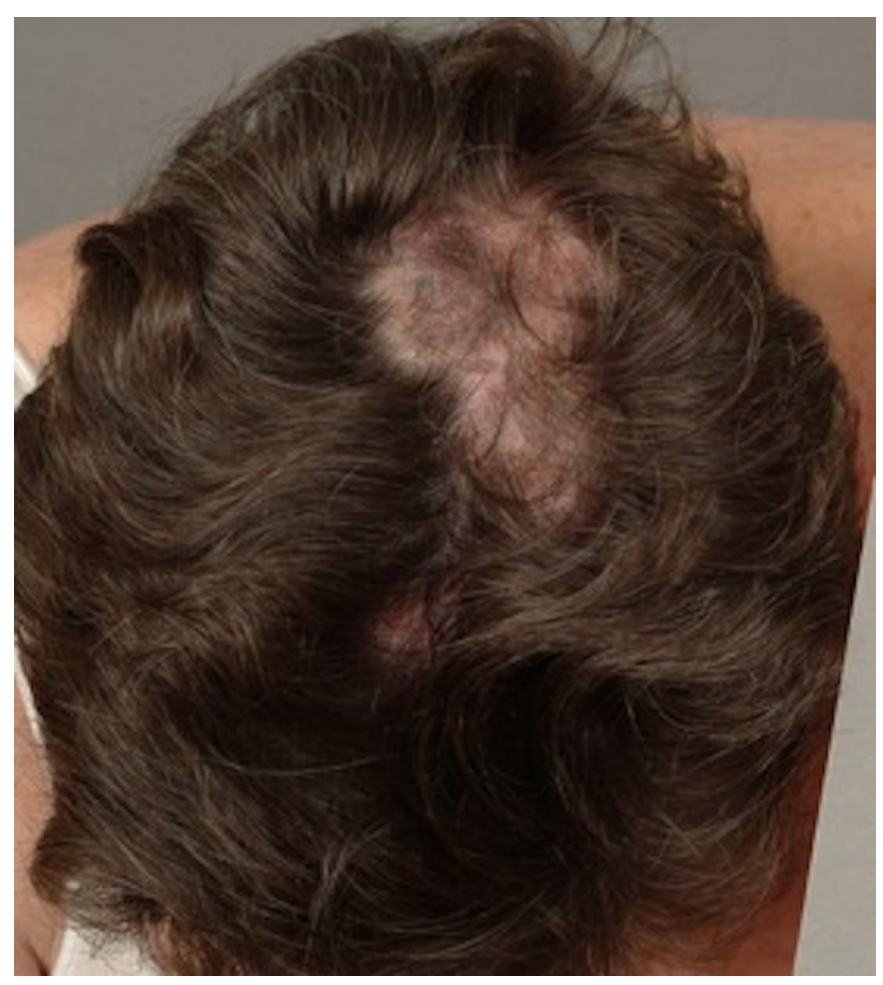

Figure 4 Subacute cutaneous lupus erythematosus. Non-scarring, scaly erythematous plaques, with similar papulosquamous lesions on the body (not depicted).

autoantibody panel in patients initially presenting with an AA-like pattern of hair loss to avoid missing the diagnosis of $\mathrm{LE} .{ }^{17}$ In contrast to $\mathrm{AA}$, once the $\mathrm{LE}$ disease activity is controlled, hair loss also improves. ${ }^{16}$

\section{Other forms of lupus-specific alopecia}

Although SCLE does not typically involve the head and neck region, ${ }^{63}$ there have been reports of SCLE affecting the scalp and resulting in patchy hair loss. ${ }^{21} 22$ These lesions are usually non-scarring, and the morphology can be polycyclic, annular or psoriasiform with thick adherent scale (figure 4). ${ }^{21} 22$ Histopathology showing psoriasiform hyperplasia with a perivascular mononuclear cell infiltrate can be similar to psoriasis; however, a positive lupus band test favours the diagnosis of SCLE. ${ }^{22}$ Distinguishing SCLE from early DLE can be challenging.

Tumid LE can rarely affect the scalp. The presentation can either be non-inflammatory round patches of hair loss similar to alopecia areata, ${ }^{20}$ or erythematous reticulated indurated plaques typical of papulonodular mucinosis. ${ }^{23}$ Biopsy shows no interface change, but displays mucinous infiltration in the deep dermis. ${ }^{20} 23$ Hair regrowth is possible with antimalarials or intralesional corticosteroids. $^{20} 23$

\section{LUPUS NON-SPECIFIC ALOPECIA}

Most non-scarring alopecias in the setting of LE occur in conditions other than LE. ${ }^{16}$ Hair loss is classified as non-scarring if, at the end of the disease process, hair growth is reversible. ${ }^{27} 66$ Follicular openings are

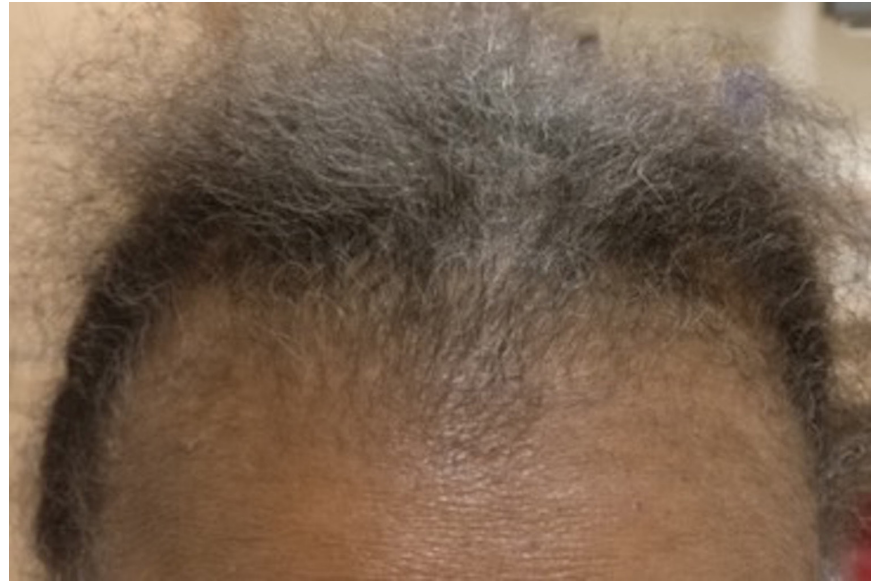

Figure 5 Lupus hair. Fine, brittle hairs on the frontal scalp in a patient with SLE.

appreciated on close-up examination of the scalp and can be further visualised with the aid of a trichoscope. ${ }^{66} 67$ Collectively, they constitute more than half of the diagnoses of LE-related alopecia. ${ }^{17}$ The diagnosis of these alopecias can be very challenging because they have no clear triggers. $^{24}$

\section{Lupus hair}

It was Armas-Cruz et $a l^{68}$ who first described the appearance of dry and fragile short hairs on the frontal hairline of patients with lupus and coined the term 'lupus hair' because this sign is so striking that it can suggest the diagnosis of SLE even by just observing a patient from afar (figure 5). The prevalence in a certain cohort of patients with SLE can range from $5 \%$ to $30 \%$, and it is found predominantly in women with chronically active SLE. ${ }^{15} 2469$ This condition is postulated to be either due to hair shaft breakage ${ }^{68}$ or to retarded hair growth, ${ }^{15}$ although a trichogram performed on a few cases showing pointed hairs supports the latter. ${ }^{69}$ The presence of this type of hair loss in a patient with SLE suggests disease activity or exacerbation, ${ }^{156869}$ oftentimes improving after the LE flare subsides. ${ }^{14}$ Growth of fine depigmented hairs can be seen in some patients during the recovery phase. ${ }^{69}$ There is no formal consensus as to what the clinical definition of 'lupus hair' should be. Some consider 'lupus hair' to be a variant of telogen effluvium, ${ }^{14} 15$ whereas others characterise it as vellus hairs on the anterior scalp. ${ }^{24}$ Moreover, there are no trichoscopic or histopathological features of this clinical sign available in the literature.

\section{Alopecia areata}

There is an increased incidence of alopecia areata in LE which reflects their similar autoimmune nature. ${ }^{70}$ Circular and completely alopecic patches, devoid of erythema or scaling are the characteristic clinical features of AA (figure 6). ${ }^{62} \mathrm{~A}$ gentle hair pull test on the edges of the patch reveals dystrophic anagen hairs. ${ }^{66}$ Dermoscopy classically shows yellow dots, black dots and exclamation point hairs. ${ }^{49} \mathrm{~A}$ mononuclear cell infiltrate is typically found surrounding the anagen bulb, although 


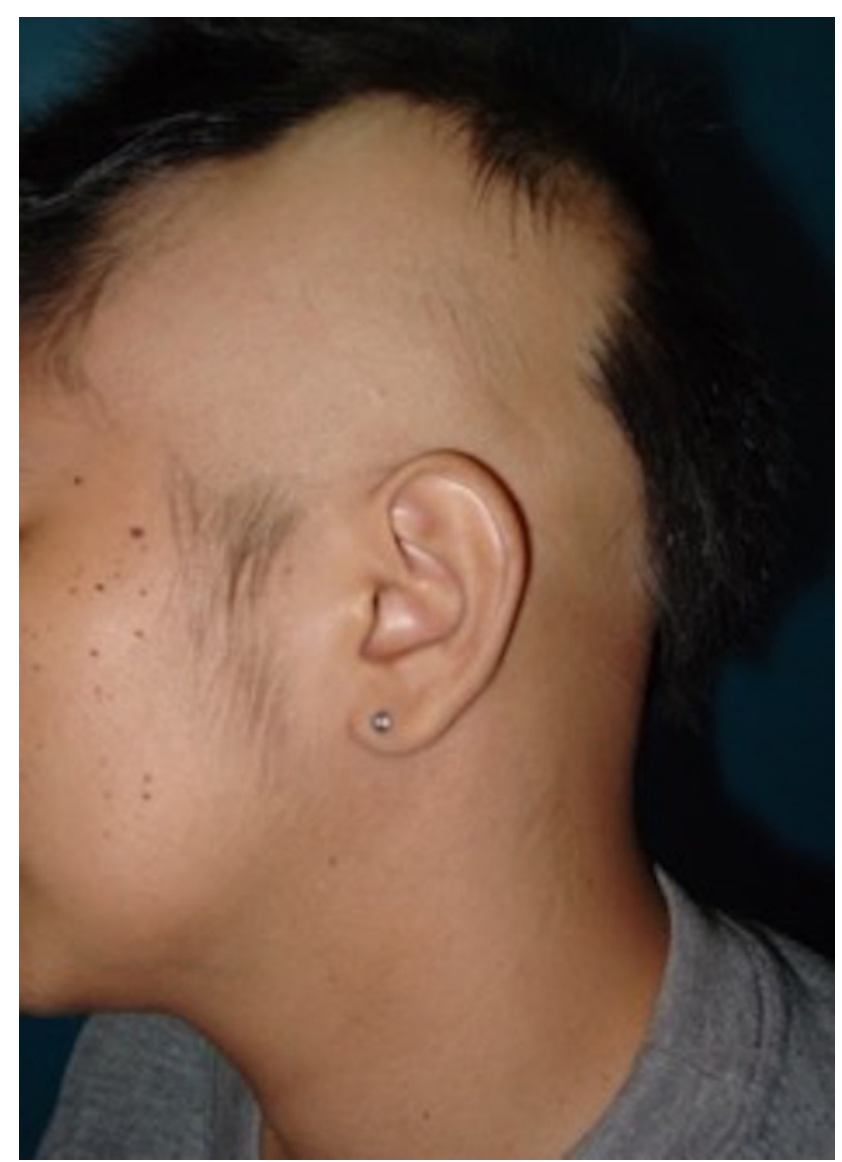

Figure 6 Alopecia areata. Non-inflammatory circular patch of hair loss on the parietal scalp.

this microscopic finding can also be found in patchy lupus alopecia and syphilitic alopecia. ${ }^{65}$ If deep dermal mucin deposition is found in addition to the usual biopsy features of alopecia areata, it might be reasonable to search for underlying SLE. ${ }^{71}$ Cases of tumid LE ${ }^{20} 23$ and lupus panniculitides ${ }^{72} 73$ have been reported to be initially diagnosed as AA, pending histopathology. The prognosis of AA is different from the patchy alopecia in LE because AA can have an unpredictable course, ${ }^{62}$ whereas the patchy hair loss in LE responds favourably to immunosuppressive therapy. ${ }^{17}$

Ophiasis is more known as a rare form of alopecia areata in which the hairless region follows a band-like distribution along the parieto-temporo-occipital region. ${ }^{17} 74$ In LE, it can be both scarring or non-scarring. ${ }^{14}$ Although it is regarded as one of the major features that portend a poor prognosis in $\mathrm{AA},{ }^{62}$ there are no available data to say this risk is the same as for those with LE.

\section{NON-LUPUS ALOPECIAS Telogen effluvium}

High fever, anaemia, sudden corticosteroid withdrawal, certain drugs, and physical or mental stress can precipitate this type of hair loss. ${ }^{11} 14-162666$ Severe metabolic breakdown and increase in certain proinflammatory markers can destructively affect the hair growth cycle. ${ }^{15}$
The hair follicles then undergo a reactive process manifesting clinically as diffuse hair shedding and/or thinning, often involving both the temporal regions. ${ }^{1466}$

Patients often complain of falling hair when they brush or run their fingers through their hair, or when they get up from bed and several strands are left on the pillow. ${ }^{13}$ Around 150-400 hairs are being shed per day, but it is not until $30 \%$ of the scalp is affected that thinning becomes noticeable. ${ }^{141626}$ A simple hair pull test, which is performed by randomly grasping around 40 hairs, will reveal a count of more than $10 \%$ telogen hairs, whereas a hair card placed perpendicularly to the scalp will demonstrate hair regrowth in the recovering disease. ${ }^{26} 66$ Trichoscopic findings are typically non-diagnostic. ${ }^{49}$ In a condition known as chronic telogen effluvium, hair loss is abrupt, severe and diffuse, with a fluctuating course lasting for more than 6 months. ${ }^{75}$

\section{Anagen effluvium}

The use of cytotoxic agents (eg, cyclophosphamide) or antimetabolites (eg, methotrexate) in LE and flares in disease activity can cause the rapidly dividing matrical cells of the anagen follicle to temporarily shut down. ${ }^{14667476}$ This abrupt arrest in anagen is clinically seen as hair shaft tapering (Pohl-Pinkus constriction), which is prone to intrafollicular breakage. ${ }^{15} \mathrm{~A}$ hair pull test will reveal several 'pencil-point hairs' and telogen hairs. ${ }^{76}$ Black dots, exclamation mark hairs and monilethrix-like hairs may be appreciated on dermoscopy. ${ }^{49}$ Timing and onset are critical because anagen effluvium classically occurs as early as 7 days after the triggering event, whereas telogen effluvium ensues months later ${ }^{2674}$; there can be an overlap between these two conditions in some patients. ${ }^{74}$

\section{Differential diagnoses of non-scarring alopecias}

Patterned hair loss

The typical location of patterned hair loss (PHL) is on the crown and frontal hairline in men, and on the midline of the crown in women (figure 7). ${ }^{26}$ A gradual course and a positive family history of PHL can rule in the diagnosis. ${ }^{66}$ Hair pull test is negative, and characteristically seen on trichoscopy is an increased number of thin, sometimes hypopigmented, vellus hairs admixed with intermediate and thick pigmented hair strands. ${ }^{4966}$ However, TE and PHL can coexist, resulting in a more pronounced presentation of alopecia. ${ }^{66}$ Moreover, PHL is a progressive disease, and therefore difficult to exclude in a patient with lupus with a history of gradually evolving diffuse hair loss (box 2). ${ }^{24}$

\section{Acute diffuse and total alopecia areata}

This recently reported variant can be clinically indistinguishable from TE. The usual clinical scenario would be a sudden and total hair loss in an older woman, without identifiable triggers and who never had a history of alopecia. ${ }^{77}$ A clue is the distribution of hair loss which can be more prominent in the temporal and retroauricular areas in diffuse $\mathrm{AA}{ }^{78} \mathrm{~A}$ hair pull test may yield 


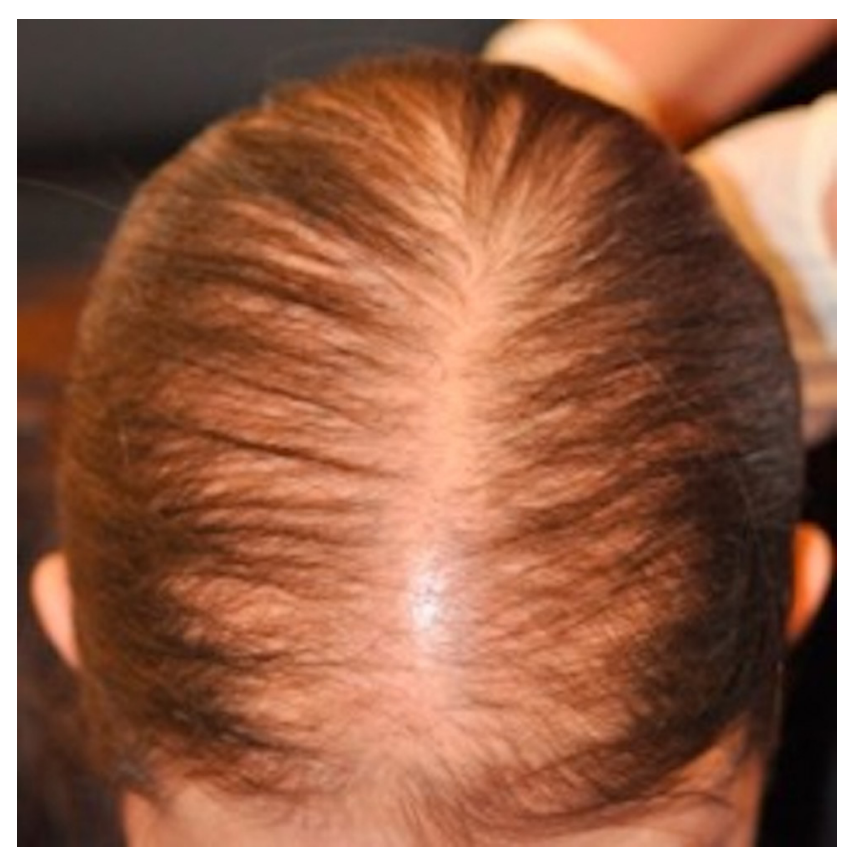

Figure 7 Patterned hair loss in a female patient. Alopecia resembles a 'Christmas tree' configuration on the crown.

dystrophic anagen hairs, but biopsy may be needed to ultimately diagnose this condition. ${ }^{62}$ Similar to AA, peribulbar monocytic infiltration and increase in telogen and catagen follicles are characteristic. ${ }^{77}$

\section{Trichotillomania}

Compulsive hair plucking can lead to broken hairs with varying lengths and bizarre skipped patches on the scalp, eyebrows or eyelashes. ${ }^{2662}$ Although the condition can be diffuse, fine vellus hairs with different lengths are more prominent on the frontoparietal or frontotemporal scalp opposite the dominant hand. ${ }^{14}$ Trichoscopy can further visualise these physical exam findings. ${ }^{49}$ Excessive and sudden tension on the hair follicles microscopically produces haemorrhages and 'pigment casts' that represent matrix cells that are torn upward and are stuck along the follicular tract. ${ }^{65}$ Absence of significant peribulbar inflammation distinguishes this from alopecia areata. ${ }^{65}$ Trichotillomania is associated with an obsessive-compulsive disorder, and with anxiety and depression, ${ }^{26}$ which are not uncommon among patients with lupus. ${ }^{79}$

\section{Syphilitic alopecia}

Alopecia can be an early manifestation of secondary syphilis and presents as 'moth-eaten' hairless patches resembling alopecia areata or diffuse hair thinning such as in telogen effluvium/anagen effluvium. ${ }^{80} 81$ It is also considered a form of anagen arrest, such as what occurs in a severe illness. ${ }^{76}$ Histopathological infiltrates can resemble those seen in alopecia areata. ${ }^{81}$ In trichoscopy, deep red-brown background pigments represent smallvessel vasculitis. ${ }^{41}$ With a high index of suspicion, a full infectious work-up (rapid plasma reagin, venereal disease research laboratory test and fluorescent treponemal antibody absorption test, among others) should be carried out. $^{81}$

\section{Tinea capitis}

Although tinea capitis is uncommon in the adult population, a chronically immunocompromised state such as in LE can predispose a patient to acquiring fungal infections on unusual sites like the scalp. ${ }^{82}$ Depending on the fungal species, the presentation can be scales, follicular erythema and broken hairs, ${ }^{66}$ or can be a greyish patch superimposed on chronic DLE lesions. ${ }^{82}$ Plucked hairs should be examined with potassium hydroxide and sent for cultures. ${ }^{14}$

\section{CONCLUSION}

Hair loss in LE can be LE-specific, non-specific to LE or coincidental with LE. Scalp DLE, the classic case of LE-specific hair loss, has a high incidence of scarring which negatively impacts the quality of life of these patients. Non-scarring alopecias can also display histopathological changes that are specific to LE. Diffuse non-scarring alopecias generally reflect the underlying systemic activity in LE. Both scarring and non-scarring alopecias can have overlapping features and can resemble non-LE causes of hair loss. The diagnosis of alopecia occurring in the setting of LE has implications on the classification of SLE. In cases where the underlying process is unclear, working with dermatologists and utilisation of dermoscopic, histopathological, immunopathological and serological data may be necessary.

Contributors JSSC and VPW have contributed equally to the manuscript. VPW is responsible for the overall content as guarantor.

Funding This work was supported by the US Department of Veterans Affairs (Veterans Health Administration, Office of Research and Development and Biomedical Laboratory Research and Development) and NIH R01AR071653 (VPW).

\section{Competing interests None declared.}

Patient consent Not required.

Provenance and peer review Commissioned; externally peer reviewed. Data statement There are no unpublished data available for this literature review.

Open access This is an open access article distributed in accordance with the Creative Commons Attribution Non Commercial (CC BY-NC 4.0) license, which permits others to distribute, remix, adapt, build upon this work non-commercially, and license their derivative works on different terms, provided the original work is properly cited, appropriate credit is given, any changes made indicated, and the use is non-commercial. See: http://creativecommons.org/licenses/by-nc/4.0

\section{REFERENCES}

1. Petri M, Orbai AM, Alarcón GS, et al. Derivation and validation of the systemic lupus international collaborating clinics classification criteria for systemic lupus erythematosus. Arthritis Rheum 2012;64:2677-86.

2. Yu C, Gershwin ME, Chang C. Diagnostic criteria for systemic lupus erythematosus: a critical review. J Autoimmun 2014;48:10-13.

3. Salehi-abari I. Autoimmune diseases and therapeutic approaches 2015 ACR / SLICC revised criteria for diagnosis of systemic lupus erythematosus. 2015;2:2-5.

4. Rothfield N, Sontheimer RD, Bernstein M. Lupus erythematosus: systemic and cutaneous manifestations. Clin Dermatol 2006;24:348-62.

5. Biazar C, Sigges J, Patsinakidis N, et al. Cutaneous lupus erythematosus: first multicenter database analysis of 1002 patients 
from the European Society of Cutaneous Lupus Erythematosus (EUSCLE). Autoimmun Rev 2013;12:444-54.

6. Grönhagen CM, Gunnarsson I, Svenungsson E, et al. Cutaneous manifestations and serological findings in 260 patients with systemic lupus erythematosus. Lupus 2010;19:1187-94.

7. Hejazi EZ, Werth VP. Cutaneous lupus erythematosus: an update on pathogenesis, diagnosis and treatment. Am J Clin Dermatol 2016;17:135-46.

8. Wysenbeek AJ, Leibovici L, Amit M, et al. Alopecia in systemic lupus erythematosus. Relation to disease manifestations. J Rheumatol 1991;18:1185-6.

9. Das NK, Dutta RN, Sengupta SR. Skin lesions in lupus erythematosus: a marker of systemic involvement. Indian J Dermatol 2011;56:537-40.

10. Hithyshi V, Dhanyasree S, Ramachandra BV. Dermatological manifestations - are they specific markers for systemic involvement in SLE? J Evol Med Dent Sci 2015;4:15749-55.

11. Kole AK, Roy R. Alopecia in lupus: experience from a tertiary referral centre. Indian J Rheumatol 2012;7:3-6.

12. Parodi A, Cozzani E. Hair loss in autoimmune systemic diseases. $G$ Ital Dermatol Venereol 2014;149:79-81.

13. Kole AK, Roy R. Alopecia in lupus: experience from a tertiary referral centre. Indian Journal of Rheumatology 2012;7:3-6.

14. Moghadam-Kia S, Franks AG. Autoimmune disease and hair loss. Dermatol Clin 2013;31:75-91.

15. Trüeb RM. Involvement of scalp and nails in lupus erythematosus. Lupus 2010;19:1078-86.

16. Bunagan MJK, Banka N, Shapiro J. Hair loss in lupus erythematosus. In: encyclopedia of medical immunology - autoimmune diseases. New York: Springer Science + Business Media, 2014: 455-9.

17. Ye Y, Zhao Y, Gong Y, et al. Non-scarring patchy alopecia in patients with systemic lupus erythematosus differs from that of alopecia areata. Lupus 2013;22:1439-45.

18. Gong $\mathrm{Y}, \mathrm{Ye} \mathrm{Y}, \mathrm{Zhao} \mathrm{Y}$, et al. Severe diffuse non-scarring hair loss in systemic lupus erythematosus - clinical and histopathological analysis of four cases. J Eur Acad Dermatol Venereol 2013;27:651-4.

19. Gong Y, Ye Y, Zhao Y, et al. Answer to: 'reply to: severe diffuse non-scarring hair loss in systemic lupus erythematosus - clinical and histopathological analysis of four cases'. J Eur Acad Dermatol Venereol 2013;27:1457.

20. Hoverson K, Jarell AD, Wohltmann WE. Lupus erythematosus tumidus of the scalp masquerading as alopecia areata. Cutis 2017;99:22-4.

21. Newton RC, Jorizzo JL, Solomon Jr MD. Subacute cutaneous lupus erythematosus. Arch Dermatol 1986;122:170-6.

22. Rai VM, Balachandran C. Subacute cutaneous lupus erythematosus (SCLE) presenting in childhood. Dermatol Online J 2005;11:27.

23. Singh AH, Werth VP. Alopecia associated with papulonodular mucinosis (tumid) lesions of lupus erythematosus. J Clin Rheumatol 1997;3:343-5.

24. Yun SJ, Lee JW, Yoon HJ, et al. Cross-sectional study of hair loss patterns in 122 Korean systemic lupus erythematosus patients: a frequent finding of non-scarring patch alopecia. J Dermatol 2007;34:451-5.

25. Özcan D, Seçkin D. Reply to: 'Severe diffuse non-scarring hair loss in systemic lupus erythematosus - clinical and histopathological analysis of four cases'. J Eur Acad Dermatol Venereol 2013;27:1456-7.

26. James W, Ti B, Elston D. Diseases of the Skin Appendages. Andrews' Diseases of the Skin. 12th edn. Elsevier, 2011: 741-82.

27. Semble AL, McMichael AJ. Hair loss in patients with skin of color. Semin Cutan Med Surg 2015;34:81-8.

28. Gilliam JN, Sontheimer RD. Distinctive cutaneous subsets in the spectrum of lupus erythematosus. J Am Acad Dermatol 1981;4:471-5.

29. Lanuti E, Miteva M, Romanelli P, et al. Trichoscopy and histopathology of follicular keratotic plugs in scalp discoid lupus erythematosus. Int J Trichology 2012;4:36.

30. Hordinsky M. Cicatricial alopecia: discoid lupus erythematosus. Dermatol Ther 2008;21:245-8.

31. Wilson CL, Burge SM, Dean D, et al. Scarring alopecia in discoid lupus erythematosus. Br J Dermatol 1992;126:307-14.

32. Chung HJ, Goldberg LJ. Histologic features of chronic cutaneous lupus erythematosus of the scalp using horizontal sectioning: Emphasis on follicular findings. J Am Acad Dermatol 2017;77:349-55.

33. Fabbri P, Amato L, Chiarini C, et al. Scarring alopecia in discoid lupus erythematosus: a clinical, histopathologic and immunopathologic study. Lupus 2004;13:455-62.

34. Rongioletti F, Christana K. Cicatricial (scarring) alopecias. Am J Clin Dermatol 2012;13:247-60.
35. Tan E, Martinka M, Ball N, et al. Primary cicatricial alopecias: clinicopathology of 112 cases. J Am Acad Dermatol 2004;50:25-32.

36. Olsen EA, Bergfeld WF, Cotsarelis G, et al. Summary of North American Hair Research Society (NAHRS)-sponsored Workshop on Cicatricial Alopecia, Duke University Medical Center, February 10 and 11, 2001. J Am Acad Dermatol 2003;48:103-10.

37. Harries MJ, Meyer KC, Paus R. Hair loss as a result of cutaneous autoimmunity: frontiers in the immunopathogenesis of primary cicatricial alopecia. Autoimmun Rev 2009;8:478-83.

38. Piette EW, Foering KP, Chang AY, et al. Impact of smoking in cutaneous lupus erythematosus. Arch Dermatol 2012;148:317-22.

39. Elman SA, Joyce C, Nyberg F, et al. Development of classification criteria for discoid lupus erythematosus: Results of a Delphi exercise. J Am Acad Dermatol 2017;77:261-7.

40. Abedini R, Kamyab Hesari K, Daneshpazhooh M, et al. Validity of trichoscopy in the diagnosis of primary cicatricial alopecias. Int $J$ Dermatol 2016;55:1106-14.

41. Tosti A, Torres F, Misciali C, et al. Follicular red dots: a novel dermoscopic pattern observed in scalp discoid lupus erythematosus. Arch Dermatol 2009;145:1406-9.

42. Nguyen JV, Hudacek K, Whitten JA, et al. The technique: a novel method for the sectioning of alopecia biopsies. J Cutan Pathol 2011;38:401-6.

43. Nieboer C. The reliability of immunofluorescence and histopathology in the diagnosis of discoid lupus erythematosus and lichen planus. Br J Dermatol 1987;116:189-98.

44. Fett NM, Fiorentino D, Werth VP. Practice and educational gaps in lupus, dermatomyositis, and morphea. Dermatol Clin 2016;34:243-50.

45. Inalöz HS, Chowdhury MM, Motley RJ. Lupus erythematosus/ lichen planus overlap syndrome with scarring alopecia. J Eur Acad Dermatol Venereol 2001;15:171-4.

46. Ankad BS, Beergouder SL, Moodalgiri VM. Lichen planopilaris versus discoid lupus erythematosus: a trichoscopic perspective. Int $J$ Trichology 2013;5:204-7.

47. Somani N, Bergfeld WF. Cicatricial alopecia: classification and histopathology. Dermatol Ther 2008;21:221-37.

48. Duque-Estrada B, Estrada BD, Tamler C, et al. Dermoscopy patterns of cicatricial alopecia resulting from discoid lupus erythematosus and lichen planopilaris. An Bras Dermatol 2010;85:179-83.

49. Mubki T, Rudnicka L, Olszewska M, et al. Evaluation and diagnosis of the hair loss patient: part II. Trichoscopic and laboratory evaluations. J Am Acad Dermatol 2014;71:431.

50. Lospinoso DJ, Fernelius C, Edhegard KD, et al. Lupus erythematosus/lichen planus overlap syndrome: successful treatment with acitretin. Lupus 2013;22:851-4.

51. del Rei M, Pirmez R, Sodré CT, et al. Coexistence of frontal fibrosing alopecia and discoid lupus erythematosus of the scalp in 7 patients: just a coincidence? J Eur Acad Dermatol Venereol 2016;30:151-3.

52. Gaffney DC, Sinclair RD, Yong-Gee S. Discoid lupus alopecia complicated by frontal fibrosing alopecia on a background of androgenetic alopecia. Br J Dermatol 2013;169:217-8.

53. Contin LA. Martins da Costa Marques ER, Noriega L. Frontal fibrosing alopecia coexisting with lupus erythematosus: poor response to hydroxychloroquine. Ski Appendage Disord2016;2:162-5.

54. Miteva M, Tosti A. Dermatoscopic features of central centrifugal cicatricial alopecia. J Am Acad Dermatol 2014;71:443-9.

55. Mirmirani P, Willey A, Headington JT, et al. Primary cicatricial alopecia: histopathologic findings do not distinguish clinical variants. J Am Acad Dermatol 2005;52:637-43.

56. Amato L, Mei S, Massi D, et al. Cicatricial alopecia; a dermatopathologic and immunopathologic study of 33 patients (pseudopelade of brocq is not a specific clinico-pathologic entity). Int J Dermatol 2002;41:8-15.

57. Shimaoka Y, Hatamochi A, Hamasaki Y, et al. Discoid lupus erythematosus exacerbated by contact dermatitis caused by use of squaric acid dibutylester for topical immunotherapy in a patient with alopecia areata. J Dermatol 2008;35:151-3.

58. Tao J, Zhang X, Guo N, et al. Squamous cell carcinoma complicating discoid lupus erythematosus in Chinese patients: review of the literature, 1964-2010. J Am Acad Dermatol 2012;66:695-6.

59. lamsumang W, Sriphojanart T, Suchonwanit P. Psoriatic alopecia in a patient with systemic lupus erythematosus. Case Rep Dermatol 2017;9:51-9.

60. Das S, Blanco G, Ahmed A, et al. Cicatricial pemphigoid of the scalp mimicking discoid lupus erythematosus. J Am Acad Dermatol 2011;65:886-7.

61. Henderson CL, Lafleur L, Sontheimer RD. Sarcoidal alopecia as a mimic of discoid lupus erythematosus. J Am Acad Dermatol 2008;59:143-5. 
62. Alkhalifah A, Alsantali A, Wang E. Clinical picture, histopathology, and pathogenesis. J Am Acad Dermatol 2010;62:177-88.

63. Haber JS, Merola JF, Werth VP. Classifying discoid lupus erythematosus: background, gaps, and difficulties. Int J Womens Dermatol 2016;2:8-12.

64. Kole AK, Ghosh A. Cutaneous manifestations of systemic lupus erythematosus in a tertiary referral center. Indian J Dermatol 2009;54:132-6.

65. Sperling LC, Lupton GP. Histopathology of non-scarring alopecia. $J$ Cutan Pathol 1995;22:97-114.

66. Mubki T, Rudnicka L, Olszewska M, et al. Evaluation and diagnosis of the hair loss patient: part I. History and clinical examination. J Am Acad Dermatol 2014;71:415.

67. Serrano-Falcón C, Fernández-Pugnaire MA, Serrano-Ortega S. Hair and scalp evaluation: the trichogram. Actas Dermosifiliogr 2013;104:867-76.

68. Armas-Cruz R, Harnecker J, Ducach G, et al. Clinical diagnosis of systemic lupus erythematosus. Am J Med 1958;25:409-19.

69. Alarcon-Segovia D, Cetina JA. Lupus hair. Am J Med Sci 1974;267:241-2.

70. Werth VP, White WL, Sanchez MR, et al. Incidence of alopecia areata in lupus erythematosus. Arch Dermatol 1992;128:368-71.

71. Goldberg LJ, Sekhri V, Bhawan J. Dermal mucin in alopecia areatatell tale sign or incidental finding? J Cutan Pathol 2008;35:757-60.

72. Kossard S. Lupus panniculitis clinically simulating alopecia areata. Australas J Dermatol 2002;43:221-3.

73. Chen YA, Chen YA, Lee JY. Linear lupus panniculitis of the scalp presenting as alopecia along Blaschko's lines: a distinct variant of lupus panniculitis in East Asians. J Dermatol 2013;39:e93.
74. Patel S, Tosti A. An overview of management of drug-induced hair and nail disorders. Clin Pract 2014;11:327-39.

75. Whiting DA. Chronic telogen effluvium. Dermatol Clin 1996;14:723-31.

76. Sperling LC. Hair and systemic disease. Dermatol Clin 2001;19:711-26.

77. Lew BL, Shin MK, Sim WY. Acute diffuse and total alopecia: a new subtype of alopecia areata with a favorable prognosis. J Am Acad Dermatol 2009;60:85-93.

78. Werner B, Mulinari-Brenner F. Clinical and histological challenge in the differential diagnosis of diffuse alopecia: female androgenetic alopecia, telogen effluvium and alopecia areata - part I. An Bras Dermatol 2012;87:742-7.

79. Achtman J, Kling MA, Feng R, et al. A cross-sectional study of untreated depression and anxiety in cutaneous lupus erythematosus and dermatomyositis. J Am Acad Dermatol 2016;74:377-9.

80. Piraccini BM, Broccoli A, Starace M, et al. Hair and scalp manifestations in secondary syphilis: epidemiology, clinical Features and trichoscopy. Dermatology 2015;231:171-6.

81. Lee JW, Jang WS, Yoo KH, et al. Diffuse pattern essential syphilitic alopecia: an unusual form of secondary syphilis. Int $J$ Dermatol 2012:51:1006-7.

82. Stiller MJ, Rosenthal SA, Weinstein AS. Tinea capitis caused by trichophyton rubrum in a 67-year-old woman with systemic lupus erythematosus. J Am Acad Dermatol 1993;29(2 Pt 1):257-8.

83. Troxell MA. Ophiasis; report of two cases. AMA Arch Derm Syphilol 1954;70:812-4 